\title{
Enhanced handover algorithm in LTE femtocells network
}

\author{
Review \\ Article \\ Mahmoud Mandour ${ }^{1}$, Fayez Gebali ${ }^{2}$, Ashraf D. Elbayoumy ${ }^{1}$, Gamal Mabrouk ${ }^{1}$ \\ ${ }^{1}$ Military Technical College, Cairo, Egypt, ${ }^{2}$ University of Victoria, Canada
}

Keywords:

Femtocell, handover, markov chain, RSRQ, RSRP

\section{Corresponding Author:}

Mahmoud Mandour, Military Technical College, Cairo, Egypt, Tel: 01005118049

Email: m_mandour78@hotmail.com

\begin{abstract}
Data rate and Coverage are the main secret factors for customer satisfaction. Today, small cell technology as femtocell can achieve the upcoming demand for higher data rate for cellular networks as well as can extend the coverage area. Femtocells are known as low cost, low power and efficient way to enhance the signal in indoor/outdoor areas with little mobile coverage.Deploying femtocells networks result in more frequent initiation of a handover $(\mathrm{HO})$ procedure. The reduction of unnecessary handovers challenges is getting more considerable while more femtocells are used in the network. We propose a novel HO algorithm to eliminate the redundant handovers in Long Term Evolution (LTE) femtocells network and to nominate the most proper target femtocell for HO among many candidates. This algorithm based on the transition probability matrix to predict the user movement using Markov Chain equations. Toward this goal, we are considering Reference Symbol Received Quality (RSRQ) and Reference Symbol Received Power (RSRP), User Equipment (UE) moving direction and in which zone the UE inside the femtocell as decision criteria.
\end{abstract}

\section{INTRODUCTION}

The 3rd Generation Partnership Project (3GPP) is a communion between groups of telecommunications standards associations, have been innovated for of $(2 \mathrm{G}$, $3 \mathrm{G}, 4 \mathrm{G}$, and $5 \mathrm{G}$ ) cellular networks, to meet the aims of high-speed data communications networks. LTE and LTE Advanced (LTEA) technologies are the most tremendous technologies for future deployment of cellular networks and wireless communication system. Basically, LTE/LTE-A supports different kinds of deployment cells in the cellular network (i.e. Macro, Micro, Pico or Femtocell), where used to circulate the coverage area depending on the area. However, (Macrocell) is the largest coverage area and used for outdoor subscribers or UEs, it is known as (eNB) in the LTE networks. So, whenever the coverage of macrocell starts decrease imply that (microcells to femtocells) founded. Actually, those are used to increase the capacity and coverage for the indoor subscribers ${ }^{[1]}$.

In mobile communication, it is renowned that the indoor mobile users are the origin of most networks traffic. In modern buildings, service providers hardly trying to provide high quality service on-the-go for indoor subscribers, but many users facing problems with poor indoor connectivity due to the signal attenuation ${ }^{[2]}$.

In mobile communication, $\mathrm{HO}$ technique can be defined as allowing the connected UE's with Evolved Node-B (eNB) to be handed-off to the next eNB without any disconnection ${ }^{[3]}$.

Femtocells are known as either Femtocell Access
Point (FAP) or Home Evolved Node-B (HeNB). FAP's, are supposed to be deployed especially (e.g. in offices, schools, universities and in shopping malls ... etc). The FAPs is connected to the service provider's backbone via a wired line such as Digital Subscriber Line (DSL) or optical fiber. Figure 1 shows an example of today's and future's deployments everywhere ${ }^{[4]}$.

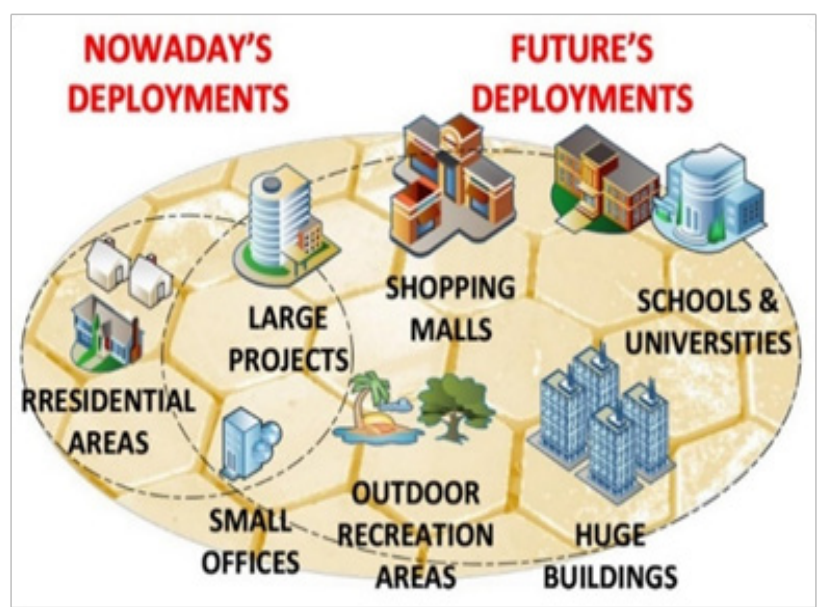

Fig. 1: Femtocell deployments networks.

There are many driving factors behind deploying LTE and LTE-A networks. One of the most important is boosting cellular network capacity by shrinking the cell size. So, the need for a technology such as FAPs is mandatory to achieve high capacity, high data rates, signal 
quality, and low latency for multimedia services indoor or in shadowed areas. Also, to increase throughput in areas with a high density of users, and to offload a large amount of capacity from the network ${ }^{[5]}$.

Recently, many studies aimed to help in user's mobility prediction. It can be classified into two schemes. One of them depends on user mobility history and the other one on user current movements. The first one, looking for a certain pattern during the user mobility in the network and creating a user mobility profile. Naturally, this approach has a complicity in mobility management, analysis, and user data updates. So, it has to pay an extra high cost for that.

This can also handle a sudden repetitive user movement. On the other hand, the second prediction approach depends on user current movement data as user speed and moving direction without considering mobility history. This one has to pay a lower cost than the user mobility history approach $^{[6]}$.

In this paper, Section II describes the related work on HO management in mobile communication networks, while Section III illustrates the background of LTE femtocells network. Section IV contains the details of modelling the system and all the assumptions. Section V, present the result.

of the study of network performance and finally, we summarize the conclusion in Section VI.

\section{RELATED WORKS}

Recent studies aimed to improve the HO performance by alleviating the unnecessary handovers and the probability of dropped calls. Also, by reducing the HO delay and reserve in advance radio resources by predicting the next handed-over target cell (i.e. either eNB or HeNB) ${ }^{[7]}$. There are many surveys shows different solutions for this issue; one of them by using prediction and creating a profile based on the history of user mobility, the other solutions based on Markov chain or based on user's location and RSRP.

$\mathrm{In}^{[8]}$, a tentative study was done to create a list with a minimum number of neighbour femtocells to start the femtocell-to-femtocell $\mathrm{HO}$ in a dense homogeneous network. The proposed method counts on the RSRP and the operating frequency of each femtocell are then defined. So, any femtocell that uses a frequency as the serving FAP is rejected.

As presented in ${ }^{[9]}$, the authors offered a Hidden Markov Model (HMM) mobility predictor way to enhance the performance of macro-cellular networks. The network deployment represents macrocells as nodes and at the edges represents the neighbouring relationships. Depend on the dynamic data of UEs and HMM, the next macrocell is identified.

The authors in $^{[10]}$, have proposed a prediction scheme depend on the history of user mobility. The network will recognize the UE whom visit the cell frequently, then track and records the movement data. Using this information and the position of the user, the network can find a route of the user and the RSRP is considered. This technique minimizes the number of HO's in LTE systems.

The work in ${ }^{[11]}$, propose a prediction technique based on the Markov Chain. This method using the path prediction to predict the target cell for the users. Position and velocity of the users are the main factors helps to expect the heading next cell for the user. In this technique, the author assumed that UE can send its location to the source cell. Simultaneously; the source cell is able to keep a database of the network coverage. Based on Markov Process, the UE movement can be predicted.

$\mathrm{In}^{[12]}$, the authors use the UE mobility history that provides frequent position and time spend on particular places. Then, using the time as an input to the transition probability matrix. After that, this matrix used to predict the UE movement by Markov Chain equations.

$\mathrm{In}^{[13]}$, a position-based path prediction scheme is used. Using a Markov Chain, the user movement has been predicted before the proper handover strategies are done.

\section{BACKGROUND OF LTE FEMTOCELL NETWORK}

Femtocells are known as a small, low-power and onthe-go base stations. It used for home/enterprise usage which provides short-range coverage over the licensed frequency band.

Femtocells offer a money-wise approach for improving the spectrum efficiency without the need to upgrade the infrastructure of the carrier network. It works as a major part of the indoor cellular network infrastructure to get a higher QoS.

Generally, LTE is designed to provide connectivity between the UE and the Packet Data Network (PDN). Figure 2 shows a simple LTE network architecture, itknown as an IP-based and flat core network.

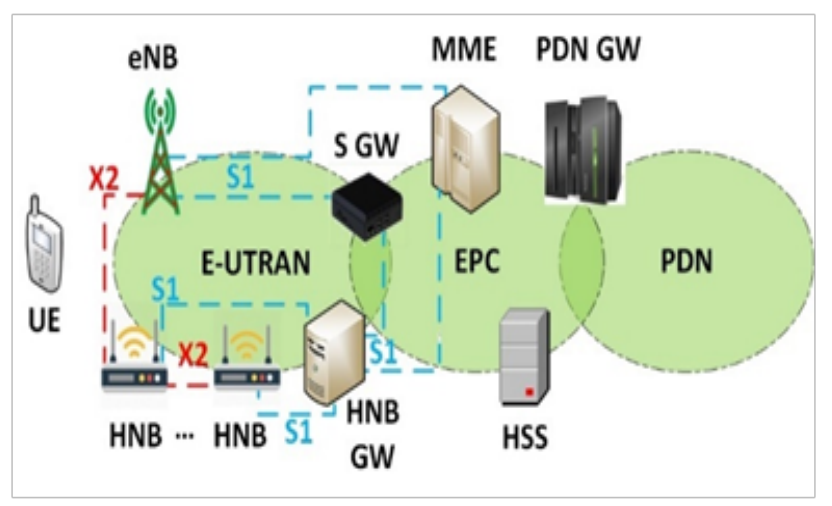

Fig. 2: LTE network architecture

There are two areas of the network at the highest level that we can consider for LTE. Those would be the (EUTRAN) which is the Evolved Universal Terrestrial Radio Access Network and the core (EPC) as the Evolved Packet Core, combined together to form the LTE network.

In E-UTRAN we encounter the eNB (macrocell) and HeNB (femtocell), which responsible to manage the air 
interface and look-after the scheduling, and handover.

The architecture deployment as a (HeNB GW) which is the HeNB Gateway, to organize the great number of HeNBs. The E-UTRAN and EPC are inter-connected by the $\mathrm{S} 1$ interface, and the eNBs/HeNBs are inter-connected by the $\mathrm{X} 2$ interface.As we move closer to the network core, in EPC we encounter the first packet core device the Serving Gateway

(S-GW). The SGW is responsible for (U-plane) which is the user plane while handovers process, also organize the data packets for the user. (i.e. it utilizes standard Internet security protocols to authorize, authenticate and encrypt the femtocells traffic between the HeNBs and the network operators core network).

Moving further to the core we encounter (PDN-GW) which is Packet Data Network Gateway, and this gives UE access to (PDN) which is the external packet data networks and to achieve high QoS we assign IP addresses to the UE.And the controlling element of the LTE network is the Mobility Management Entity (MME), This device looks after mobility management and session management. (i.e. it is responsible for control plane functions (C-plane). Supporting the MME with (HSS) which is Home Subscriber Server, acting as a central database which contains all related user and subscription information.

The HeNB shall acts like eNB for all function supported and the process run between the EPC and the $\mathrm{HeNB}^{[14]}$.

\section{A. LTE Femtocell Handover Scenarios}

There are different handover scenarios in the femtocell cellular network:

a) Hand-in: represents the UE connection is handed over from the eNB to the HeNB.

b) Hand-Out: represents the handover performed from the HeNB to the eNB.

c) Inter-HeNB: Similar to Hand-in scenario. It represents the handover done between the HeNBs.

\section{B. The Handover Types}

As shown in figure 3 LTE/LTE-A networks are supporting two different types of $\mathrm{HO}$ schemes:

a) Horizontal $\mathrm{HO}$ (homogeneous): used in the $\mathrm{HO}$ between the same type of network cells.

b) Vertical HO (heterogeneous): used in the HO between different types of network cells ${ }^{[15]}$.

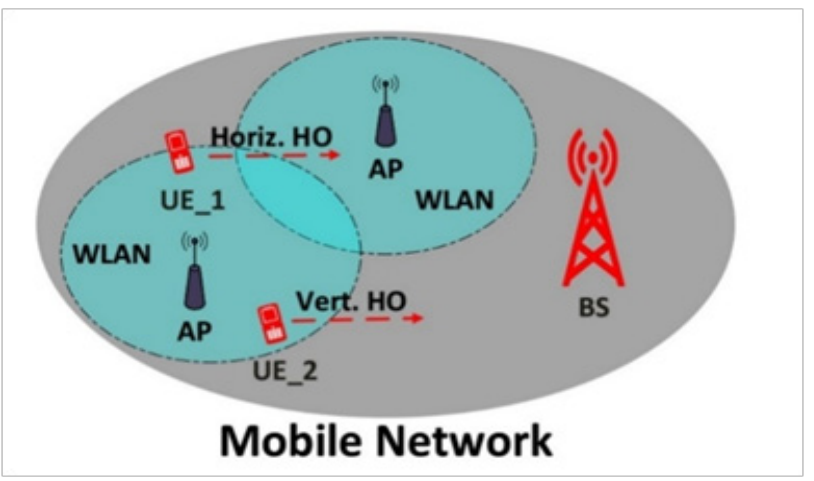

Fig 3: Handover types (vertical and horizontal)
HO mechanism should accept mutation between the homogeneous and heterogeneous of cellular networks, and also should be incorporated with different radio access networks (RAN), such as (LTE, WiMAX, WiFi, UMTS, WLAN, ... etc $)^{[16]}$.

Moreover, all information of RSRP/RSRQ related to UE and network cells are gathered for the HO triggering mechanism. Then, this data is used to evaluate the next hop (target) cells in the taking decisionphase. After choosing it, a fresh link is established in the HO execution phase where all resources are transferred from the served cell to the target cell ${ }^{[17]}$.

\section{Handover Decision}

Basically, The HO procedure can be divided into four parts:

a) Obtaining Measurement Reports from neighbour FAPs to the serving cell.

b) Appropriate Target FAP Selection.

c) HO Decision.

d) HO Execution and Completion.

Handover decision is one of the major parts of the call flow, while the handover process is done. The handover decision depending on some common factors which come from the measurement report provided by the UE. The main parameters are:

a) RSRQ represents the Reference Signal Received Quality.The reporting range is defined starting from $\geq-5 \mathrm{~dB}$ (Excellent) to $\leq-20 \mathrm{~dB}$ (No signal).

b) RSRP which is the Reference Signal Received Power. The reporting range is defined starting from

$\geq-75 \mathrm{dBm}$ (Excellent) to $\leq-120 \mathrm{dBm}$ (No signal).

We use them both to select the most appropriate target cell for the running call. First, RSRQ gives more information about how much the interference level is.

Furthermore, the more favourable is RSRQ than RSRP in the case of dense HeNB's network, which has a high interference level. In LTE network, the UE should be updated every $200 \mathrm{~ms}$ with parameters on the reference signal RSRP/RSRQ ${ }^{[18]}$.

\section{SYSTEM MODEL FOR MOBILITY AND HANDOVER}

Generally, we should discuss how the conventional (classical) HO occurred. The classical HO scheme for wireless and mobile networks show up two styles of threshold area in the coverage of the cell. The handover threshold and the exit threshold can be imagined as two different hexagons with an irregular radius around the cell core. The inner hexagon with small radius refers to the exit threshold hexagon, and the outer hexagon with larger radius refers to the handover threshold hexagon ${ }^{[19]}$.

Nevertheless, the outer threshold hexagon is the beginning of the HO process. So, for complete a successful mobility, any UE has to end the handover process before finishing the handover threshold hexagon area. If the UE is not handed-over efficiently before this threshold area, the 
UE call will be blocked (i.e. dropped) ${ }^{[20]}$.

We consider our proposed system shown in figure 4, as a homogeneous cellular network consists of many merged femtocell stations and the UE is moving randomly inside the network. Where every UE inside the network has a unique ID and neighbours depending on the UE serving cell and its type (corner/edge).

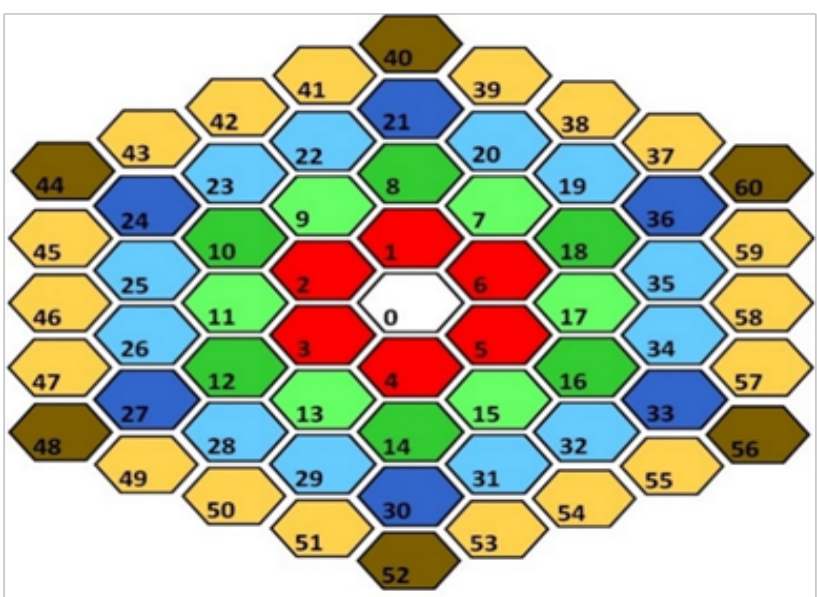

Fig. 4: Geometry of hexagonal grid for cellularfemtocell network

Figure 4 shows the architecture of our cellular femtocell network, which has rings around the central hexagon. The darker tone represents a corner hexagon, and the lighter tone represents an edge hexagon.

In network modelling, we employ the following schema:

1) We assume $60 \mathrm{FAPs}$, the numbering sequence follows a" spiral" path such that the numbers of hexagons per ring are multiple of 6 , e.g. the first ring (1-6), the second ring (7-18), the third ring (19-36), and the last ring (37-60). 2) Two FAPs can be adjacent by being neighbours along the spiralling path indicated above, in which case their IDs are also consecutive. In this spiralling counting pattern, we call the FAPs such as $7,19,37, \ldots$ (gate cells) where the path jumps to an outer larger circular ring.

3 ) We assumed that we have 6-directions rounded counter clockwise with the first ring $(\mathrm{R}=1)$ which labelled as direct-1 for cell ID 1 to the direct-6 for cell ID 6.

4) The main direction is known as the Top direction which has the IDs of $(1,8,21,40, \ldots$ etc $)$.

5) In figure 4 , all hexagons were labelled using a single non-negative integer ID, $0 \leq \mathrm{ID} \in \mathrm{Z}$; or, equivalently, using the ring number $\mathrm{R}, 0 \leq \mathrm{R} \in \mathrm{Z}$, and the user site within the ring $S, 0 \leq S \in Z$, where:

$$
\text { I D }= \begin{cases}0, & R=0 \\ 3 R(R-1)+1+S, R \geq 1\end{cases}
$$

or inversely,

$$
R=\left\{\begin{array}{lr}
0, & I D=0 \\
\lfloor((3+\sqrt{(12 * I D-3))} / 6)], & I D \geq 1
\end{array}\right.
$$

where, $\downarrow$ denote rounding towards zero, and

$$
S=I D-3 R(R-1)-1
$$

6) All characteristics with general forms of the proposed hexagonal FAPs network are listed in the table (1).

Table 1: Characteristics of hexagonal femtocells

\begin{tabular}{lccccc}
\hline Ring & \#edge cells persector & \#cells onring & Start ID & End ID & Top Corner ID \\
\hline 0 & 0 & 1 & 0 & 0 & 0 \\
1 & 0 & 6 & 1 & 6 & 1 \\
2 & 1 & 12 & 7 & 18 & 8 \\
3 & 2 & 18 & 19 & 36 & 21 \\
4 & 3 & 24 & $:$ & $:$ & 40 \\
$\mathrm{R}$ & $:$ & $:$ & $:$ & $:$ & $:$ \\
$:$ & $:$ & $6 \mathrm{R}$ & $3 \mathrm{R}(\mathrm{R}-1)+1$ & $3 \mathrm{R}(\mathrm{R}+1)$ & $\mathrm{R}(3 \mathrm{R}-2)$ \\
\hline
\end{tabular}

7) Each hexagon is divided into two different regions, the Core region where $(\mathrm{P}=0)$ and the HO region where $(\mathrm{P}=1)$. 8) At $t=t s$, when a moving UE located in the Core region that implies the serving cell and target cell will be the same. Only the UE position will change from Core to $\mathrm{HO}$ region. 9) A special case at ring $R=0$, there is only one corner hexagon (the white hexagon in the center). It has six hexagons neighbours are located in $\operatorname{ring} \mathrm{R}=1$.
10) Ring $R=1$, is kind of special because it consistsof only corner hexagons, but it turns out the samerules to handle this ring as well as all outer rings just fine.

11) All rings except ring $R=0$ has $(R-1)$ edge hexagons between consecutive corner hexagons. Particularly, each outer ring has one more edge hexagon between corner hexagons; which allows compensating the $\mathrm{P}$ indexing along the ring and in any other rings. 
12) UE located inside any ring, where $R=(0)$ to $(R-1)$ have 6-neighbours, but in the proposed solution we decrease them to only 3-neighbours based on other parameters as UE position inside the source cell (Core region/HO region) hexagon and its direction of movement.

13) As shown in figure 4, if the user located in the outer ring, first we have to check the station type (will be discussed in Algorithm 1) either (corner/edge).

14) Whenever UE moving towards the boundaries (outer ring) in any direction inside the network (i.e. Not Valid destination cell) which located in the outer ring, the moving direction will be laterally inverted, such as mirrorlike reflection. The outer the ring acts Specular Reflection and no $\mathrm{HO}$ has done in this case.

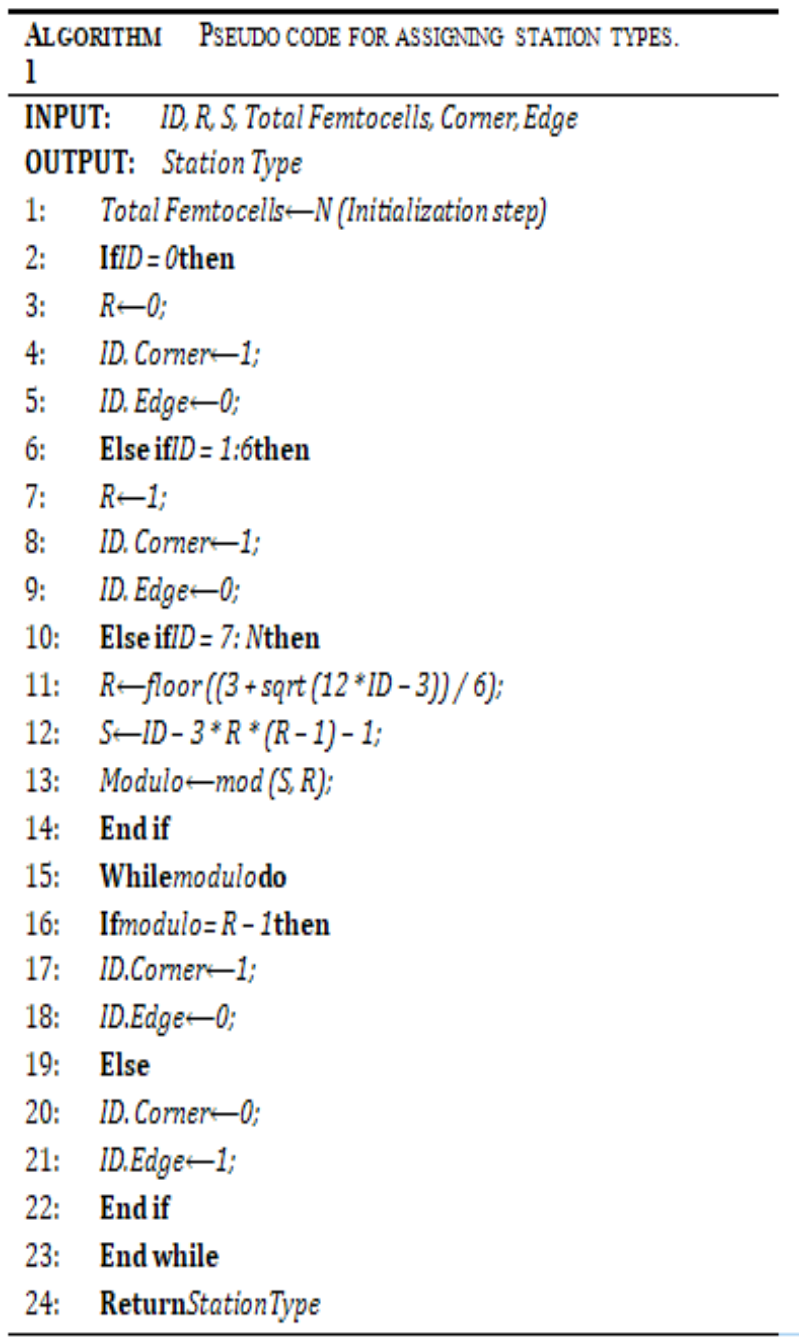

Algorithm 1 shows the proposed pseudo-code for the algorithm to minimize target FAP by assign station type in the network. We define the input parameters as (ID, R, S, $\mathrm{N}$, Corner, and Edge), an output parameter is the Station Type. For the user being served by outer edge cell, it has 4-neighbours and only 3-neighbours for the one being servedby an outer corner cell. After knowing the station type fromAlgorithm 1, we need to investigate the UE's mobility insidethe network and find-out a schema to know the neighbourcells. Mobility Checker will be described in flow-chart toillustrate when the UE needs HO or not.

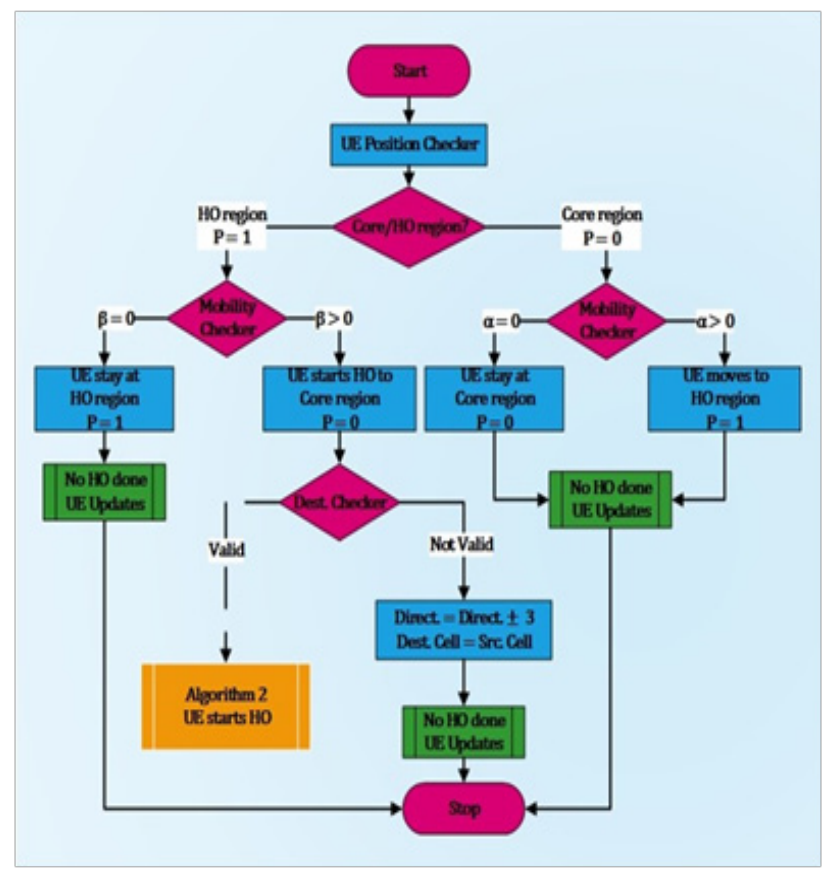

Fig. 5: Mobility checker flow control procedure for femtocells networks

Figure 5 shows how the proposed Mobility Checker flow chart works. Basically, start with checking the UE position inside the serving cell either in the Core region or HO region. After that, the Mobility checker starts based on the values of $(\alpha)$ and $(\beta) .(\alpha)$ is the transition probabilityof any UE moves inside the serving cell fromthe Core region to $\mathrm{HO}$ region. $(\beta)$ is the transition probability of any UE moves inside the serving cell fromthe $\mathrm{HO}$ region outgoing to the next core region in the target cell.

The first case, if UE located in the Core region $(\mathrm{P}=0)$, then after that we need to check the mobility $(\alpha)$ if UE is moving or not. So, the moving UE will change its position forward to HO region in the same serving cell at $(\alpha>0)$ and for the stationary UE will stay at the Core region where $(\alpha=0)$. For the second case, when UE located in HO region $(\mathrm{P}=1)$, the algorithm checks the mobility $(\beta)$. Here, the stationary UE will stay as is in $\mathrm{HO}$ region $(\beta=0)$ but for the moving one $(\beta>0)$ will be prepared to start the $\mathrm{HO}$ process as well as in algorithm 2 to finalize the $\mathrm{HO}$ procedure.

Figure 6 shows the femtocells network being studied in details, where all femtocells are modelled as a hexagon shape, overlapped coverage and with a HeNB located at the center. Figure 6(A), shows the general geometry of the femtocells under study (central blue hexagon) and one of the six neighbouring femtocells (top blue hexagon). Figure 6(B), shows the two adjacent femtocells that will be explained in details. 


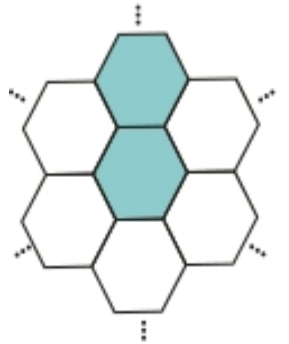

A

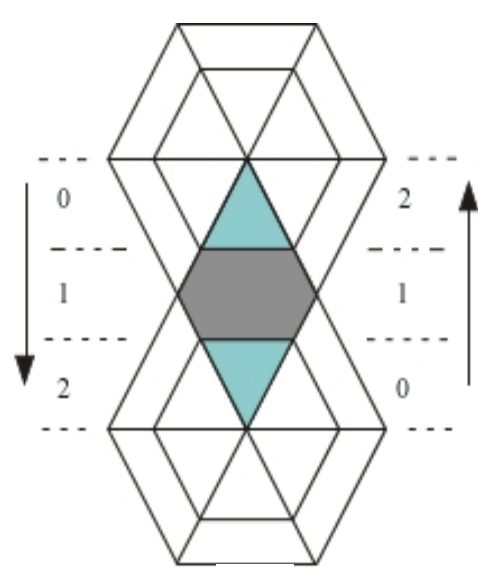

B
Fig. 6: Proposed LTE femtocells cellular network. (A) Network structure. (B) Detailed coverage areas of two neighbour femtocells

We employ the following assumptions:

1) We assume that every two neighbouring femtocells are divided into three zones, we use subscripts $(0,1$, and 2$)$ to indicate the femtocell zones in both (outgoing/incoming) directions as:

- Zone 0 represents the core of the source femtocell.

- Zone 1 represents the edges between the source femtocell and neighbouring target femtocell at the top. (i.e. HO region in grey colour).

- Zone 2 represents the core of the target femtocell.

2) Each femtocell with its neighbours has six identical queues located in $\mathrm{HO}$ region, one queue per each hexagonal sector.

3) We consider that there are many users with active calls or not. They are allocated inside the core of HeNB and the moving UE considered to be handed over from the served HeNB to the next target HeNB. (i.e. passing form (zone 0 to zone 2) direction or vice versa).

As depicted in figure 6(B), we investigate the queue exist in the HO region (grey hexagons area) between the two neighbour femtocells. Queuing analysis is one of the most important methods for studying wireless communication systems. It helps to get the truth of many inquiries about the system performance.

The basis of the approach is to propose a novel HO scheme that enhances the performance of the LTE femtocells network and optimizing the HO process. While, the quantity of the users in the queue based on its immediate past state, so our system can be modelled using Markov chain Models. Mm/Mm/1/B queue type (MultipleBirth Multiple-Death) is used. So, at any time step t, most multiple callscould arrive and at most multiple calls could leave. (i.e. thequeue size, at each time step $t$ can increase by more thanone, and also decrease by more than one) ${ }^{[21]}$.

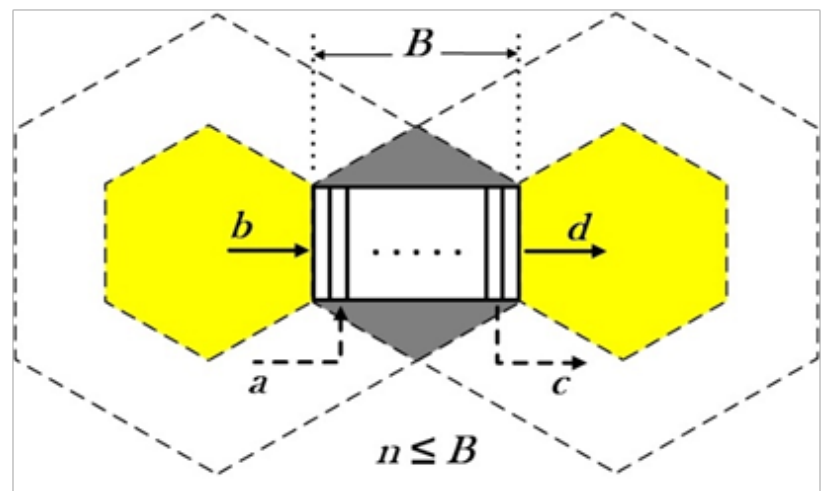

Fig. 7: The queue in handover region between two neighbour femtocells.

Figure 7 shows the two neighbour femtocells with a queue in the grey handover region.

Our proposed algorithm considering two types of calls: the incoming calls have two types are (a, b), and the outgoing calls have two types are (c, d).

The number of users per sector will represented as $n$. The queue size B can define as the waiting area for the UE as long as they have not been served yet, also the UE that is being served.

In figure 7 , we employ the following simplifying assumptions:

1) Each femtocell is further divided into two parts, the core, and the edge areas. The core area is in yellow colour, and the edge area where merged or overlapped with the target neighbour HeNB is in grey colour.

2) We use a column stochastic matrix that operates sequentially and it represents the transitioning from one state to another.

3) The incoming call types defined as:

- a represents the probability of a new call initiated inside the handover region, which is equivalent to a birth event or an increase in the queue population.

- b represents the probability of incoming call comes from the core of serving HeNB to be handed over to the target HeNB. Also, we consider the probability of no incoming call enters the queue is $(1-b)$.

4) The probabilities of $k$ calls started, are given by:

$$
P_{a}(n, k)=\left(\begin{array}{l}
n \\
k
\end{array}\right) a^{k}(1-a)^{n-k}
$$

5) And, we have two different types of outgoing calls leaving the queue in the handover region. These two types of outgoing calls are defined as:

- c represents the probability of that new call initiated (a), is terminated. So, it is known as an outgoing terminated call.

-) d represents the probability of that incoming call(b) comes 
from the core of serving HeNB, is successfully handed over to the target HeNB. So, it isknown as an outgoing handed-over call. Also, we consider the probability of no outgoing $\mathrm{HO}$ call leaves the queue is $(1-d)$.

6) The probabilities of $\mathrm{k}$ calls ended, are given by:

$$
P_{c}(n, k)=\left(\begin{array}{l}
n \\
k
\end{array}\right) a^{k}(1-a)^{n-k}
$$

7) We assume, $n \leq B$ to warrant that the number of incoming users would not exceed the queue size $\mathrm{B}$, where $\mathrm{n}$ is the number of users per sector.

8) As a basic condition, if the number of users $n$ is greater than the queue size B would imply the next user will lose the call (Blocked Calls).

9) Also, we assume:

- $N$ represents the total number of user's per HeNB.

- $k$ represents the number of active calls.

We assume that, whenever $n<k<0$, or $n<0$;

$$
\begin{gathered}
P_{a}(n, k)=0 \\
P_{c}(n, k)=0
\end{gathered}
$$

Markov Chain is a mathematical system that bears a transition process from one state to another state. Moreover, it defined as a memory-less random process. (i.e. at time step $(t+1)$, the value of the random variable depends only on its immediate past value at time step $t$ ).

Markov Chain is a transition system has $n$ states. and, the dimension of the transition probability matrix $\mathbf{P}$ will become $(n * n)$.

So, the general form of transition probability matrix $\mathbf{P}$ will be given by:

$$
\begin{aligned}
& \mathbf{P} \\
& =\left[\begin{array}{ccccc}
p_{i, 0} & p_{0,1} & \cdots & p_{0, n-1} & p_{i, n} \\
p_{1,0} & p_{1,1} & \cdots & p_{1, n-1} & p_{1, n} \\
\vdots & \vdots & \vdots & \vdots & \vdots \\
p_{n-1,0} & p_{n-1,1} & \cdots & p_{n-1, n-1} & p_{i, n} \\
p_{n, 0} & p_{n, 1} & \cdots & p_{n, n-1} & p_{n, n}
\end{array}\right]
\end{aligned}
$$

In the state transition matrix $\mathrm{P}$, each column shows the present state and the rows shows the next state. Element $p i, j$ will show the transition probability between the two states (i.e. from the initial state $\mathrm{j}$ to the final state i). For example, p0, 1 considered as the probability of the system makes a change from the present state 1 to the next state 0 . The general equations of the matrix can be written as below: At $j=0$; the first column of the transition probabilities matrix $\mathbf{P}$ as:

$$
P_{i, 0}=(1-b) x_{1}+(b) x_{2}
$$

Where,

$$
\begin{aligned}
& x_{1}=p_{a}(n-j, i) * p_{c}(0,0), 0 \leq i \leq n \\
& x_{2}=\left\{\begin{array}{lr}
p_{a}(n-j, i-1) * p_{c}(0,0), & 0 \leq i<n \\
p_{a}(n-j, i-1) * p_{c}(0,0)+ & i=0 \\
p_{a}(n-j, i) * p_{c}(0,0), & i=0
\end{array}\right.
\end{aligned}
$$

At $0<j<n$; the inner columns of the transition probabilities matrix $\mathbf{P}$ as:

$$
\begin{aligned}
& P_{i, j}=(1-b)(1-d) y_{1}+(1-b) d y_{2}+ \\
& b(1-d) y_{3}+b d y_{4}
\end{aligned}
$$

Where,

$$
\mathrm{y}_{1}=\sum_{k=0}^{j} p_{a}(n-j, k+i-j) * p_{c}(j, k), 0 \leq i \leq n
$$

$$
y_{2}= \begin{cases}\sum_{k=0}^{j} p_{a}(n-j, k+i-j+1) * p_{c}(j, k), 0<i \leq n \\ j & \\ \sum_{k=0} p_{a}(n-j, k-j+1) * p_{c}(j, k)+ & \\ p_{a}(n-j, 0) * p_{c}(j, j), & i=0\end{cases}
$$

$$
y_{3}= \begin{cases}\sum_{k=0}^{j} p_{a}(n-j, k+i-j-1) * p_{c}(j, k), 0 \leq i<n \\ j & i=n\end{cases}
$$

$$
\mathrm{y}_{4}=\sum_{k=0}^{j} p_{a}(n-j, k+i-j) * p_{c}(j, k), 0 \leq i \leq n
$$

At $j=n$, the last column of the transition probabilities matrix $\mathbf{P}$ as:

$$
\begin{aligned}
& P_{i, n}=(1-b)(1-d) z_{1}+(1-b) d z_{2}+ \\
& b(1-d) z_{3}+b d z_{4}
\end{aligned}
$$

Where,

$$
\begin{aligned}
& \mathrm{z}_{1}=p_{a}(n-j, n-j) * p_{c}(n, n-i), 0 \leq i \leq n \\
& z_{2}=\left\{\begin{array}{lr}
p_{a}(n-j, n-j) * p_{c}(n, n-i-1), & 0<i \leq n \\
p_{a}(n-j, n-j) * p_{c}(n, n-1)+ & \\
p_{a}(n-j, n-j) * p_{c}(n, n), & i=0
\end{array}\right.
\end{aligned}
$$


$z_{3}=\left\{\begin{array}{lr}p_{a}(n-j, n-j) * p_{c}(n, n-i+1), & 0<i \leq n \\ p_{a}(n-j, n-j) * p_{c}(n, 1)+ & \\ p_{a}(n-j, n-j) * p_{c}(n, 0), & i=n\end{array}\right.$

$\mathrm{z}_{4}=p_{a}(n-j, n-j) * p_{c}(n, n-i), 0 \leq i \leq n$

$\mathrm{d}_{r}=\sum_{j=0}^{n} \mathrm{~s}(\mathrm{i})[(1-\beta) / 2] *(1-1 / \mathrm{j})$

Mobility prediction helps to discover which cell will be the next (target) cell to finish the handover procedure. In this paper, Markov Chain predictor is used to predict the target femtocell for the moving handed-over user. The predictor uses the transition probability matrix $\mathrm{H}$, where, the values of $\mathrm{H}$ are derived from the Markov Chains state diagram and the highlighted circles illustrate the different states being studied.

Our model described as a set of different states $(u 0, u 1$, $u 2, u 3)$. A set of transitions; where every single transition represents a movement from one state to another state.

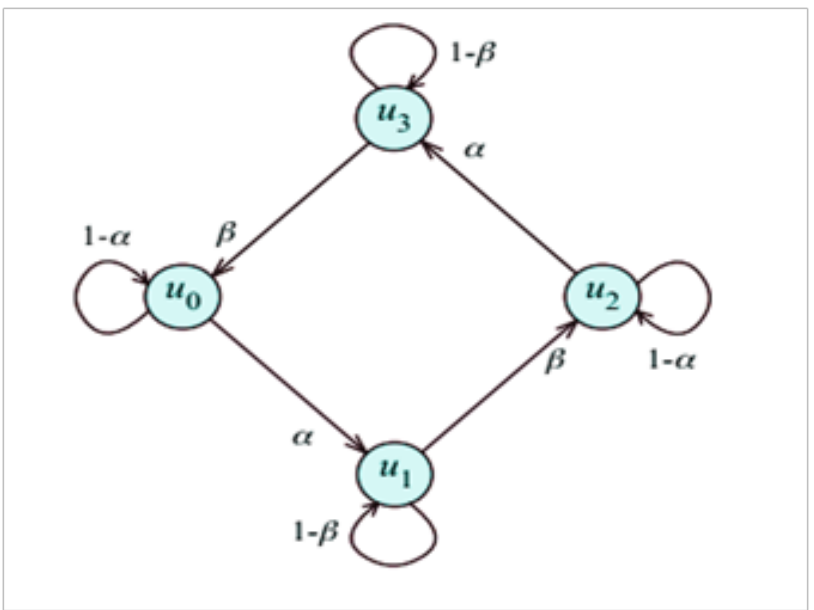

Fig. 8: Markov chain state transition diagram for femtocell system

Figure 8 shows the state transition diagram for thefemtocell system, where the transition probabilities $\alpha$ and $\beta$ depend on the deployment of the femtocells and theUE's mobility inside the network. We can define these mainparameters as:

1) $\alpha$ represents the normalized velocity of any UE moves from the core of serving femtocell outgoinghandover region.

2) $\beta$ represents the normalized velocity of any UE moves to outgo from the handover region to thecore region in the target femtocell.

The transition probability matrix $\mathrm{H}$ is developed from Markov Chain state diagram. In our proposed scenario, the UE will have three assigned probabilities in every two neighbour femtocells. So, the UE will move from state to the next state where, $(u 0)$ and $(u 2)$ represents the core of serving and target HeNB's, respectively and ( $u 1$, u3) represents the merged edges between the neighbours serving/target HeNB's (i.e. the two femtocell base stations under investigation). Every column represents the serving HeNB, while the row represents the target HeNB.

$$
\mathbf{H}=\left[\begin{array}{cccc}
(1-\alpha) & 0 & 0 & \beta \\
\alpha & (1-\beta) & 0 & 0 \\
0 & \beta & (1-\alpha) & 0 \\
0 & 0 & \alpha & (1-\beta)
\end{array}\right]
$$

Basically, $\alpha$ and $\beta$ are selected to ensure a positive column-stochastic matrix. It means that the summation of each column is equal to 1 , means the summation of $(\alpha)$ and $\mathrm{m}(1-\alpha)$ is equal to 1 ; and the same concept for the other columns.

We apply our matrix equations to MATLAB to solve it as:

$$
\begin{aligned}
& u_{0}=(1-\alpha) u_{0}+\beta u_{3} \\
& u_{1}=\alpha u_{0}+(1-\beta) u_{1} \\
& u_{2}=\beta u_{1}+(1-\alpha) u_{2} \\
& 1=u_{0}+u_{1}+u_{2}+u_{3}
\end{aligned}
$$

By using the Symbolic Math Toolbox, we get:

$$
\begin{aligned}
& u_{0}=\left(\frac{\beta}{\alpha}\right) u_{3} \\
& u_{1}=\left(\frac{\alpha}{\beta}\right) u_{0} \\
& u_{2}=\left(\frac{\beta}{\alpha}\right) u_{1} \\
& u_{3}=\left(\frac{\alpha}{\beta}\right) u_{2}
\end{aligned}
$$

Figure 9 shows how the HO decision phase has done. Starting with the information comes from the Measurement Reports from neighbour FAPs to the serving cell.

Towards the paper aim, we have three steps to nominate 
the most proper target FAP among many candidates and to eliminate the redundant $\mathrm{HO}$ in the proposed cellular network.

First, if the (RSRP and RSRQ) of the target cell > thresholds of serving cell it will pass to the second filter; if not, the target cell will be rejected.

After-that, if the target Cell load Capacity $<$ Cell threshold Capacity (assumed $85 \%$ ) it will pass to the third filter; if not, the target cell will be rejected. The last filter examines the available BW of the target cell.

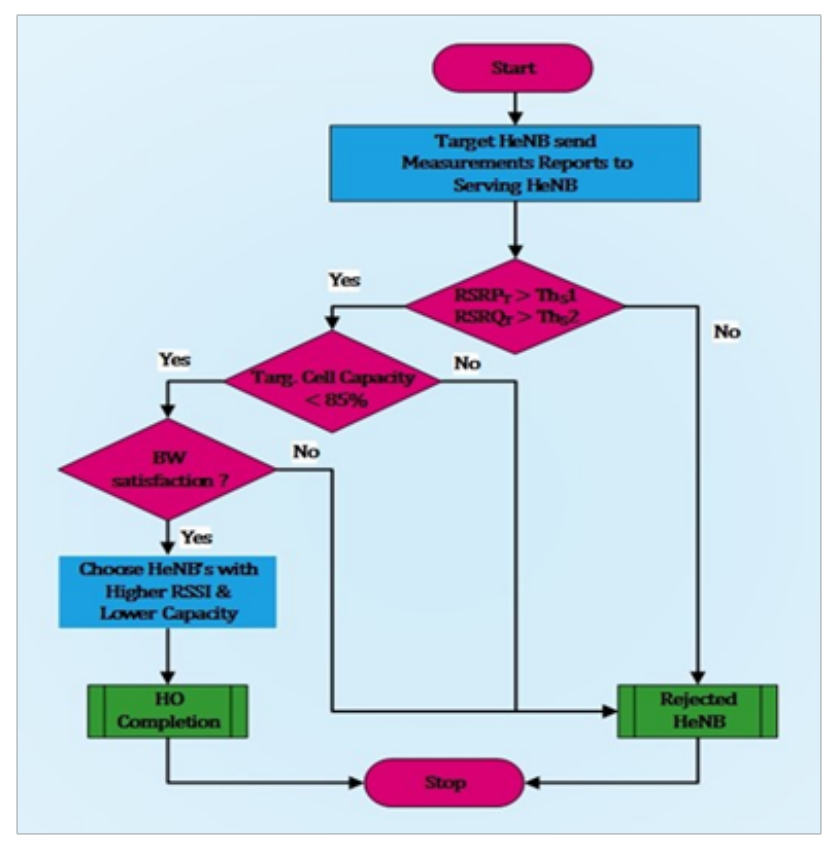

Fig. 9: Handover decision procedure for femtocells networks

\begin{tabular}{|c|c|}
\hline \multirow{2}{*}{\multicolumn{2}{|c|}{ 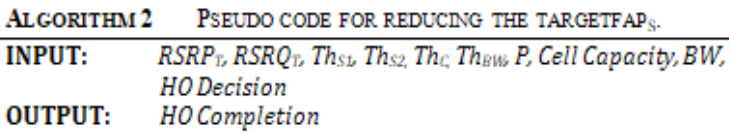 }} \\
\hline & \\
\hline 1: & $R S R P_{T}, R S R Q_{T} \leftarrow$ ? (Signalmeasurements) \\
\hline 2: & If $R S R P_{T}>T h_{S t}$ then \\
\hline 3: & $P \leftarrow 1$; FAPselection $)$ \\
\hline 4: & Else \\
\hline 5: & $P \leftarrow 0 ;($ Rejected FAP $)$ \\
\hline 6: & End if \\
\hline 7: & If $R S R Q_{T}>T h_{S 2}$ then \\
\hline 8: & $P \leftarrow 1 ;($ FAPselection $)$ \\
\hline 9: & Else \\
\hline 10: & $P \leftarrow 0 ;($ Rejected FAP $)$ \\
\hline 11: & End if \\
\hline 12: & IfCell Capacity < Thc then(LoadCapacity) \\
\hline 13: & $P \leftarrow 1 ;($ FAPselection $)$ \\
\hline 14: & Else \\
\hline 15: & $P \leftarrow 0 ;($ Rejected FAP $)$ \\
\hline 16: & End if \\
\hline 17: & Loop:(ResourceAvailability) \\
\hline 18: & IfBW $<T_{B}$ uthenreturn (Best Target FAP Selected) \\
\hline 19: & HO Decision $\leftarrow 1 ;$ \\
\hline 20: & IfBW $>T h_{B}$ then (Reject selected FAP\& take thenextone) \\
\hline 21: & HO Decision $\leftarrow 0$; \\
\hline 22: & Gotoloop; \\
\hline 23: & Close; \\
\hline 24: & ReturnHOCompletion \\
\hline
\end{tabular}

The pseudo-code in Algorithm 2 shows in steps how to arrange all survived target cells according to their RSRP/ RSRQ values, lower load Capacity and BW requirements of the UE are satisfied.

Now, UE authorized to access one of the survived targetsFAP which has the higher signal strength. FAP loadcapacity for the connected UE'sshould not exceed $85 \%$ of themaximum allowed number.

By applying this concept, the source FAP can easily selectthe appropriate target FAP and can achieve our goal ofavoiding a greater number of unsuccessful $\mathrm{HO}$.

\section{PERFORMANCE ANALYSIS}

In this section, the performance of the proposed network is analyzed using MATLAB simulation tool. The most relevant simulation parameters are listed in Table (2). We evaluate the performance for the proposed algorithms in terms of user mobility in HO region with Success/Failure HO probability.

Table 2: Simulation parameters list

\begin{tabular}{lc}
\hline Parameter & Value \\
\hline Simulation Time & $10-500(\mathrm{sec})$ \\
Simulation Area & $200 \times 200(\mathrm{~m})$ \\
Cellular Layout & 61 (FAPs) \\
$\#$ UE's/FAP & $1-10(\mathrm{UE})$ \\
UE direction & Randomly $(1-6)$ \\
Gate Cells & $7,19,37$ \\
Cell Capacity & Max 10 (UE's) /FAP \\
$\alpha$ and $\beta$ & $0-1$ \\
\hline
\end{tabular}

Hence, the proposed algorithm based on adding more $\mathrm{HO}$ decision metrics.For taking the HO decision we considerthe level ofRSRP and RSRQ from the target FAPs. Moreover, the level of RSS indicatoralso used which is the mainly reference effective value whilethe traditional process occurred. Furthermore, adding another feature of network weighty to give flexibility to the service provider for HO decision based on the Capacity of FAP to minimize the effect of ping-pong compared with the traditional scheme.

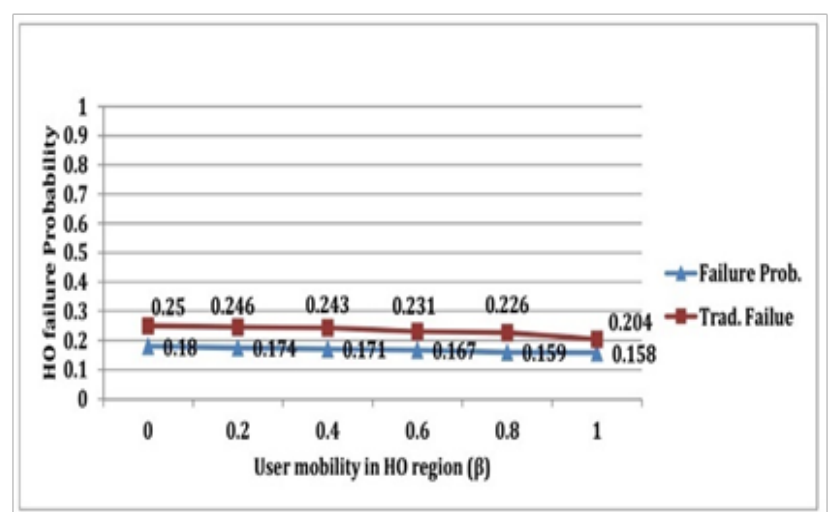

Fig. 10: HO failure probability 
Figure 10 shows a comparison between the proposed and the traditional schemes, the traditional mechanism has a little bit higher HO failure rate than the proposed one, because it is only based on the (RSSI) Received Signal Strength Indicator.Considering that the reporting range is defined starting from $>-65 \mathrm{dBm}$ (Excellent) to $\leq$ $-95 \mathrm{dBm}$ (No signal).

However, the proposed mechanism reduces the HO failure probability because it considers different factors such as signal strength, cell capacity, and the BW availability.

Note that, by deploying a large number of FAPs in the network, it will not make the HO failure probability rise. So, the HO failure rate of the proposed scheme will decrease as long as increasing the number of deployed FAPs.

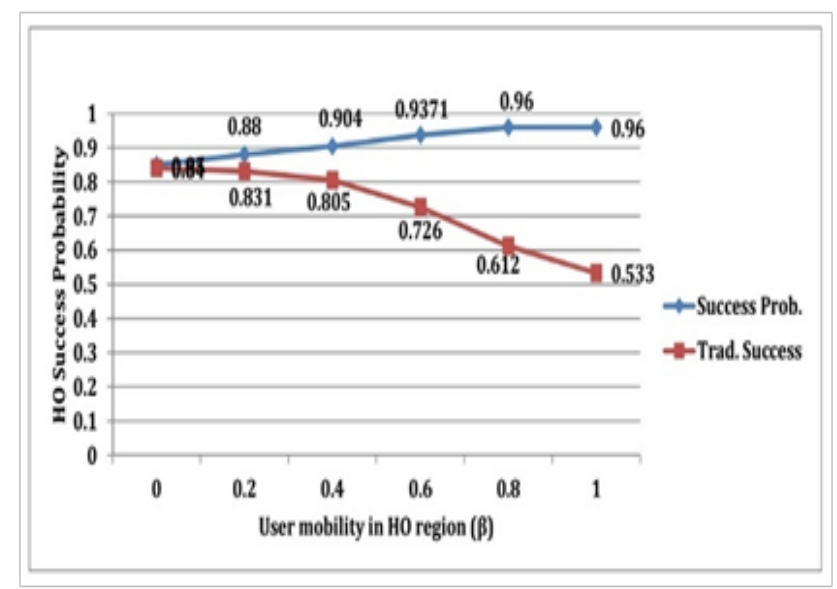

Fig. 11: HO success probability

Figure 11 shows the HO success probability for the survived target FAPs increased ; consequently, we are using only the survived target FAPs for HO process.Considering figure 12 , the previous two figures were combined in one figure with different format to make easy comparison for the reader.

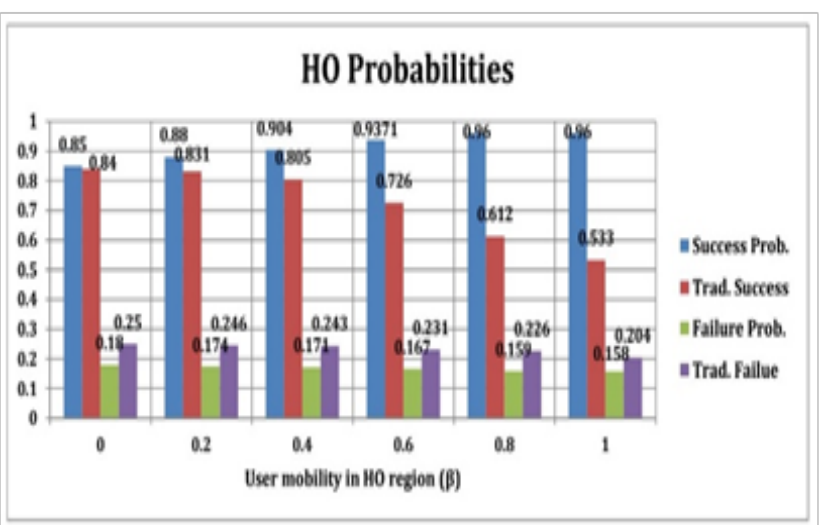

Fig. 12: The entire network HO probability

Figure 12 shows the overall $\mathrm{HO}$ probability in the entire network compared with the traditional scheme.

\section{CONCLUSION}

In this paper, the simulation result shows up some advantages of the new proposed $\mathrm{HO}$ mechanism as eliminating the unnecessary $\mathrm{HO}$ and ensure the load balance of the target FAP and the entire network.

Also, the serving cell takes into consideration the real capacity of the available survived target FAP. Note that, if the target FAP has a large UE capacity state around the threshold, the serving FAP will give up and will choose the next lighter load cell for the HO process.

Finally, this mechanism can lighten the over-charge of resources usage by using the BW resources of selected target FAP only.

The results demonstrate that using the proposed scheme to predict the best target FAP for $\mathrm{HO}$ willintroduce a better performance compared to the traditional procedure.

By the way, it improves the QoS in the entire network by decreasing the failure probability rate and reducing the effect of ping-pong with an almost stable level.

\section{REFERENCES}

[1] R. Ahmad, E. A. Sundararajan, and N. E. Othman, "Handover in LTE-advanced wireless networks: state of art and survey of decision algorithm," Springer Telecommun Syst, vol. 66, no. 16, Mar. 2017.

[2] A. M. Miyim, M. Ismail, and R. Nordin, "Vertical handover solutions over LTE-advanced wireless networks: An overview," SpringerWireless Pers Commun, vol. 52, no. 30, Mar. 2014.

[3] A. Hasbollah, N. F. S. H. S. Ariffin, M. Ismail, and R. Nordin, "Mobility prediction method for vehicular network using markovchain," JurnalTeknologi, vol. 52, no. 2, Jun. 2016.

[4] T. V. T. Duong and D. Q. Tran, "An effective approach for mobility prediction in wireless network based on temporal weightedmobility rule," International Journal of Computer Science and Telecommunications, vol. 3, Feb. 2012.

[5] H. A. Salman, L. F. Ibrahim, and Z. Fayed "Overview of LTE-advanced mobile network plan layout," in IEEE 5th InternationalConference on Intelligent Systems, Modelling and Simulation, no. 27, Jan. 2014.

[6] S. H. S. Ariffin, E. A. Sundararajan, N. N. N. A. Malik, and N. E.Ghazali, "Mobility prediction via markov model in LTE femtocell,"International Journal of Computer Applications, vol. 65, no. 18, Mar.2013.

[7] A. Ulvan, R. Bestak, and M. Ulvan, "Handover procedure anddecision strategy in LTE-based femtocell network," Springer Telecommun Syst, vol. 52, no. 9, Sep. 2011

[8] M. Z. Chowdhury, B. M. Trung, and Y. M. Jan, "Neighbour celllist optimization for femtocell-to-femtocell handover in dense femtocellular networks," in IEEE Third International Conference onUbiquitous and Future Networks (ICUFN), no. 15, Jun. 2011.

[9] H. SI, Y. WANG, J. YUAN, and X. SHAN, "Mobility predictionin cellular network using hidden markov model," in IEEE 7thIEEE Consumer Communications and Networking Conference, no. 9, Jan. 2010.

[10] H. Ge, X. Wen, W. Zheng, Z. Lu, and B. Wang, "A history-basedhandover prediction for LTE systems," in IEEE InternationalSymposium on Computer Network and Multimedia Technology, Jan.2009, p. 14.

[11] A. Ulvan, M. Ulvan, and R. Bestak, "The enhancement of handoverstrategy by mobility prediction in broadband wirelessaccess," in NAEC Networking and Electronic Commerce ResearchConference, vol. 55 , no. 6 , Oct. 2009.

[12] Z. H., W. X., W. B., Z. W., and Y. Sun, "A novel handover mechanism between femtocell and macrocell for LTE based networks,"in Second international conference on communication software andnetworks, vol. ICCSN'10, no. 228231, Jul. 2010.

[13] K. T., Y. Q., L. J., P. S., and Y. Shin, “A mobility managementtechnique with simple handover prediction for $3 \mathrm{~g}$ LTE systems," inIEEE 66th vehicular technology conference, vol. VTC-2007, no.259263,Mar. 2007. 
[14] G. T. V13.2.0, “Technical specification group radio access network; evolved universal terrestrial radio access (E-UTRA) andevolved universal terrestrial radio access network (E-UTRAN);overall description," Springer Telecommun Syst, vol. Stage 2, no.Release 13, Jan. 2016.

[15] J. M. Barja, C. T. Calafate, J. C. Cano, and P. Manzoni, "Anoverview of vertical handover techniques: Algorithms, protocolsand tools," Elsevier Computer Communications, vol. 34, no. 1, Jun.2010.

[16] Y. Zhou and B. Ai, "Ahandover schemes and algorithms of highspeed mobile environment: A survey," Elsevier Computer Communications, vol. 47, no. 1, Jul. 2014.

[17] V. Yazc, U. C. Kozat, and M. O. Sunay, "A new control plane for5g network architecture with a case study on unified handoff, mobility, and routing management," IEEE Communications Magazine,vol. 52, no. 21 , Nov. 2014.
[18] P. G., S. B., R. S., S. X., and K. S., "A novel approach for mobilitymanagement in LTE femtocells," IJWMN International Journal of Wireless and Mobile Networks, vol. 6, no. 5, Oct. 2014.

[19] Y. Kirsal, E. Ever, A. Kocyigit, O. Gemikonakli, and G. Mapp, "Analytical modelling of a new handover algorithm for improveallocation of resources in highly mobile environments," Springer, The Journal of Supercomputing, vol. 71, no. 1, Oct. 2015.

[20] Y. K. Ever, Y. Kirsal, E. Ever, and O. Gemikonakli, "Analyticalmodelling and performability evaluation of multi-channel WLANS withglobal failures," International Journal of Computer Communicationsand Control, vol. 10, no. 1, Aug. 2015.

[21] F. Gebali, “Analysis of Computer Networks", Second Edition. New York:Springer, 2015. 